\section{Cooperation in Social Insects}

Raghavendra Gadagkar

Centre for Ecological Sciences,

Indian Institute of Science, Bangalore, India

Many species of insects, such as ants, bees, wasps and termites, organize themselves into societies that parallel, and sometimes better, human societies. They have impressive levels of social organization, communication, division of labor, cooperation and conflict, altruism and self-sacrifice, policing and punishment, and learning and teaching. They can accomplish feats in warfare with neighboring colonies or feats of internal cooperation such as the construction of sophisticated nests or mounds, which are impossible for individual colony members. They are organized into colonies whose sizes range from a few individuals up to a million or more, occupying from a few centimeters to hundreds of square kilometers (Fig. 1).
Consider a honey bee colony - perhaps the best known insect society (Winston 1987; Seeley 2010; Page 2013). Their colonies comprise tens of thousands of individual bees, among which there is a single, large, and fertile female bee who is called the queen. Only the queen is mated, and with a store of sperm gathered from many males during her nuptial flight at the beginning of her life, she can lay thousands of eggs per day, both fertilized eggs that develop into females and unfertilized eggs that develop into males. Colonies may comprise a few male bees, also known as drones. The proverbially lazy drones do not take part in social life and do not contribute to domestic work. Instead, they leave their nests of birth and attempt to mate with virgin queens of other colonies. Drones successful in mating die in the act of copulation as their genitals are severed from their bodies and left hanging onto the females. The rest of the colony comprises smaller nearly sterile female bees, called workers. Workers have lost their genitalia over evolutionary time and cannot mate and cannot produce 


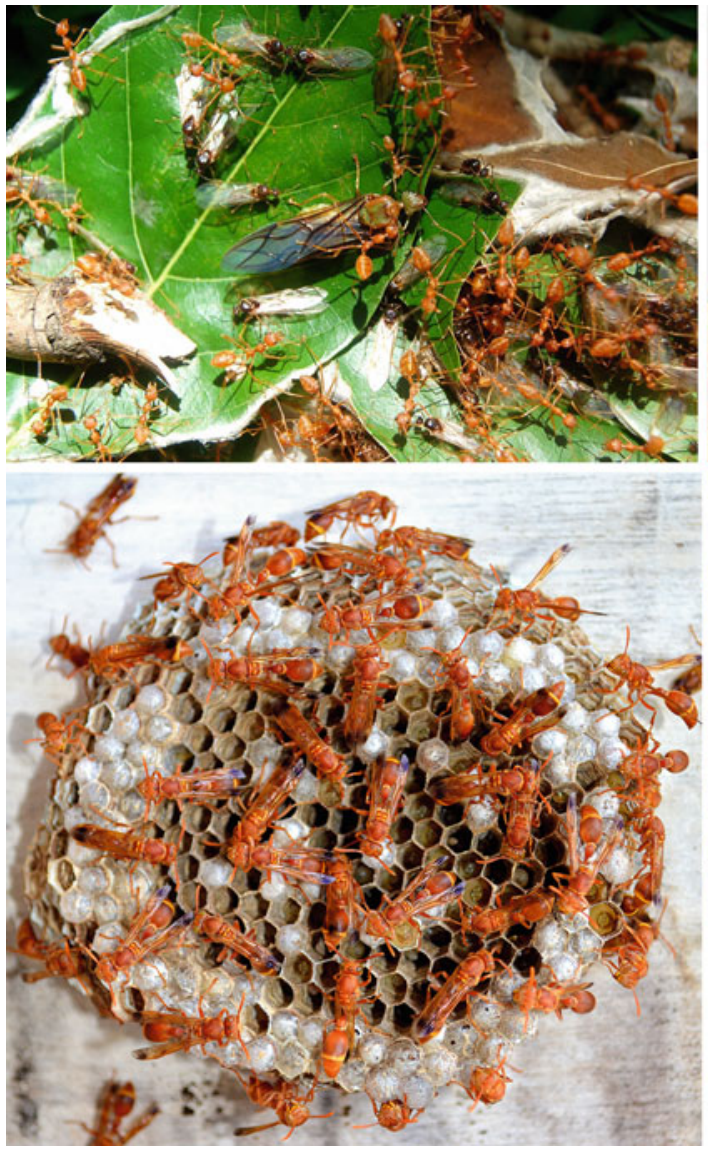

Cooperation in Social Insects, Fig. 1 Examples of the major eusocial insects. Upper left, the tropical weaver ant, Oecophylla smaragdina; upper right, the Asian dwarf honey bee Apis florea; lower left, the best studied Indian

female offspring. They have small ovaries (much smaller than those of the queen), but these usually remain undeveloped in the queen's presence. Only upon the death of the queen and the colony not being able to rear a new queen do some workers develop their ovaries and lay a few haploid, male-destined eggs. In the presence of a healthy queen, workers refrain from reproduction and spend their whole lives working for the welfare of their colonies. Various tasks required for the functioning of the colony are performed in an
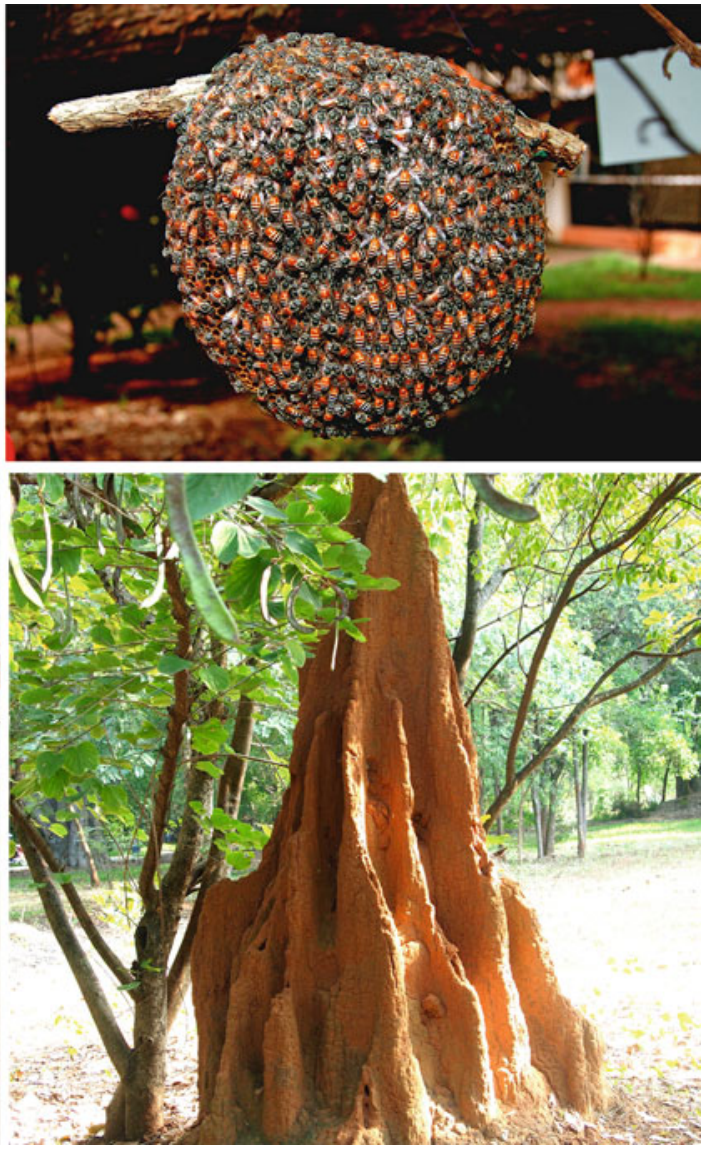

paper wasp Ropalidia marginata; lower right, the tropical mound-building termite (Odontotermes obesus). (Photos courtesy Dr. Thresiamma Varghese)

orderly, systematic fashion through a process known as age polyethism. Young bees begin by cleaning the nest, after which they successively transition to perform the tasks of building the nest, feeding the larvae, guarding the nest, and finally venturing out to forage for pollen and nectar, which serve as the sources of protein and carbohydrate, respectively, for the growing larvae. The task a bee performs depends on its relative age in the colony. At the age at which they build the nest, they have active wax glands that convert 
the nectar consumed into wax, which is used for building the nest. As the worker bees get older, these wax glands degenerate, and their hypopharyngeal glands develop instead, secreting, many substances that are added to the diet of the larvae. Such relative age-based polyethism has a built-in flexibility permitting the colony to adaptively respond to unexpected changes in demography. Upon experimental removal of old bees, the relatively older of the intranidal workers becomes precocious foragers, leaving the nest at an abnormally younger absolute age than expected in normal colonies. Conversely, upon experimental removal of young bees, the youngest of the foragers become over-aged nurses, reverting to nursing, regenerating their already atrophied hypopharyngeal glands.

Division of labor is also modulated by the spatial distribution of tasks, and of the bees, and by their relative sensitivities of to the cues signaling the need to perform certain tasks. Queens mate with several males and produce daughters of several patrilines, contributing to the variability in the sensitivities of the bees to perform different tasks. For instance, bees differ in their response thresholds to the smell of dead bees so that undertaker bees are those that are most sensitive to the smell. If a colony is missing the most sensitive patriline, however, the intensity of the smell will soon be adequate for another patriline to remove the dead bees. Sources of pollen and nectar are located by some foragers, acting as scout bees; successful scouts return to the hive and recruit naïve bees to transport the pollen or nectar back to the hive. This they do by the well-known honey bee dance language. When the source is nearby, they perform a round dance motivating the bees to search nearby for the flowers with the expected smell. When the source is more than about $500 \mathrm{~m}$, they perform a waggle dance, communicating specific information about the distance and direction of the food source. Bees estimate the distance and direction of the food source by the image motion on their eyes of the surrounding landscape (optic flow) and a process of dead reckoning called path integration, keeping track of their successive linear and angular displacements as they meander on their outward search flights (Wehner 1992, Mandal 2018). Worker bees thus devote their whole lives to the welfare of their colony and do not reproduce, even in the form of a few male-destined eggs that they can potentially lay. This is an extreme level of cooperation that we label as altruism. Even more impressive is their readiness to sting any marauder of their nest, an act that leads to their immediate death, as they cannot withdraw their stings from the bodies of their victims, owing to its outwardpointing barbs.

While there are some 10 species of honey bees worldwide, all belonging to the genus Apis, in the family Apidae, there are at least 15,000 species of ants, all belonging to the family Formicidae, found everywhere except in the Arctic and Antarctic poles. The ants have reached comparable levels of social complexity. Their species diversity permits the ants to embrace many lifestyles (Hölldobler and Wilson 1990). For instance, leaf-cutting ants practice a sophisticated form of agriculture, cultivating fungi in their gardens and feeding a population of up to a million or more individuals only with the products of their labor - fungal spores. Medium-sized workers go out, locate, cut, and transport leaves back to their nests. These pieces of leaves of carefully chosen species are then ground into a fine paste and spread in the garden by even smaller workers. Fungal mycelia are then "planted" in the garden, and the spores eventually harvested. The fungi cultivated - certain kinds of mushrooms - are not known to exist outside the fungal gardens of the ants. Certain cultivars of the 
fungi appear to have been selected and passed down across generations of ant colonies and occasionally exchanged between colonies. The ants have elaborate adaptations for manuring their gardens with their feces and for keeping out weeds, with the help of a cocktail of antibiotics produced by bacteria they harbor on their own bodies.

In contrast, weaver ants dwell in nests on trees built by stitching leaves together with silk threads. While hundreds of ants use their combined effort to bend and maneuver leaves in alignment, some of them squeeze well-grown larvae to persuade them to donate some of their silk for the community good. Such donation of silk normally meant for their own pupation is costly to the larvae and constitutes an act of altruism even before the individual becomes an adult. Reminiscent of the lazy drones in honey bees, male ant larvae are less likely to contribute silk for nest building. Weaver ants are voracious predators in the tropical habitats of Africa (Oecophylla longinoda) and Asia (Oecophylla smaragdina) and build interconnected series of nests across several trees, with a single queen producing all the eggs required to maintain their colony populations.

In even greater contrast, ants of the genus Diacamma in Asia have permanently lost their queens. Their relatively small colonies (of a few hundred individuals) consist only of workers, one of whom is mated and functions as a gamergate (mated worker) and lays all the eggs for their colony. All workers are born with a pair of wing buds called gemmae, which appear to be necessary for the female ants to attract males. The gamergate physically mutilates the gemmae of all eclosing workers and thus prevents them from mating and becoming reproductive. If the gamergate dies, the next worker to eclose retains her gemmae, becomes a gamergate, and mutilates all those who eclose after her. Mutilated workers perform all the tasks such as nest building, brood care, and foraging, required to rear the brood of the gamergate. Diacamma genus presents a unique method of reproductive regulation, leading to monogamy, i.e., reproductive monopoly by one egg-laying individual.

All social wasps are paper wasps because they construct their nests not with wax as honey bees do, not with leaves as some ants do, and not with soil as termites do but with paper. They manufacture their own paper by scraping cellulose fibers from plants, adding their own secretions to make a pulp and drying it. All social wasps belong to the family Vespidae. There is a very wide variation in the level of social evolution within the Vespidae. Species belonging to the subfamily Vespinae display advanced sociality resembling the honey bees, while species belonging to the subfamily Stenogastrinae are socially very primitive, bordering on the solitary. The subfamily Polistinae are intermediate and are considered primitively eusocial (see below). Their queens and workers are not morphologically differentiated, and most adults eclose being capable of taking on both queen and worker roles. Polistine wasps have been especially useful in investigating the evolution of social behavior because they display the transition from solitary to social life within the lifetime of individual wasps. New polistine wasp nests may be founded by a single-mated female who can, much like a solitary insect, build and guard her nest, lay eggs, forage, and bring her offspring to adulthood, all by herself. Her daughters from the first brood often stay back as sterile workers and assist her in producing more offspring, making the nest now a social unit. New nests may also be founded by small groups of females, in which case they are social units from the start. Female wasps may function as workers for some time and then transition to a queen status by leaving their parent nests and starting new single foundress nests and new multiple foundress nests as their queens or may replace the queens of their parental nests. These transitions permit us to study 
the costs and benefits of solitary as well as social life and understand the ecological forces that mold the evolution of social behavior, despite the cost of non-reproduction (Gadagkar 2001).

Termites represent the fourth main group of insect societies. They are rather different in many ways. First, they do not belong to the insect order Hymenoptera like the ants, bees, and wasps but belong to a different insect order, the Isoptera. Second, unlike the Hymenopteran social insects which are haplodiploid (see below), they are diploid. They have both queens and kings that live together as a monogamous pair. Their workers are also of both sexes. In some species, the immature nymphs serve as workers and never complete their metamorphosis into adults. Workers ingest plant material, digest the cellulose with the help of gut microbiota, and feed the larvae and even the adult soldiers by regurgitating food to them by a process known as trophallaxis (Korb and Thorne 2017).

High levels of cooperation have made the social insects ecologically successful - in some tropical forest habitats, social insects, especially, termites and ants, may constitute three-quarters of the animal biomass. Biologists often use the terms cooperation and altruism interchangeably. In both cases, the reference is to behavior costly for the actor and beneficial to the recipient. As described above, social insects are replete with examples of such costly cooperation. However, their levels of social integration and division of labor vary greatly between different species so that they may be labelled as communal, quasi-social, para-social, semi-social, sub-social, or eusocial (Wilson 1971). The last label, "eusocial," is considered the pinnacle of the evolution of social complexity. Eusocial species exhibit (i) cooperative brood care, so that individuals routinely care for offspring not their own; (ii) overlap of generations, so that caregivers can be younger than those who receive care; and (iii) reproductive division of labor, so that only one or a few individuals in the colony reproduce, called queens or kings as the case may be, while the rest function as sterile helpers, often called workers. Among the eusocial species, it is customary to recognize two further sub-divisions, namely, the primitively eusocial and the advanced or highly eusocial. Until the 1970 s, eusociality was thought to be restricted to ants, bees, and wasps in the insect order Hymenoptera and termites in the order Isoptera. In the last few decades, eusociality has also been discovered in thrips in the order Thysanoptera, aphids in the order Hemiptera, ambrosia beetles in the order Coleoptera, and shrimps, in the arthropod class Crustacea. Barring the case of the naked mole rat that lives in underground tunnels in Africa which curiously fits the definition, eusociality is restricted to insects and some shrimps. Despite this taxonomic range expansion of eusociality, advanced eusociality is restricted to ants, bees, wasps, and termites (Hölldobler and Wilson 1990; Gadagkar 2001; Rubenstein and Abbot 2017).

Biologists ask two kinds of questions about biological traits, the so-called proximate questions (sometimes called "how" questions) and ultimate questions (sometimes called "why" questions). In the context of cooperation in social insects, examples of the proximate kind include questions such as how do social insects recognize nestmates versus non-nestmates and maintain colony integrity; how do they divide colony labor among themselves; how does each member of the colony know what work to do when, and what not to do, so that they can function as an effective group; and how do they accomplish complex group tasks such as building elaborate nests, of locating, bringing, and processing food; etc. There is one overarching ultimate question, namely, how does natural selection favor the evolution of altruistic sterility by a process that is supposed to bring about survival of the fittest (fitness being usually measured as the 


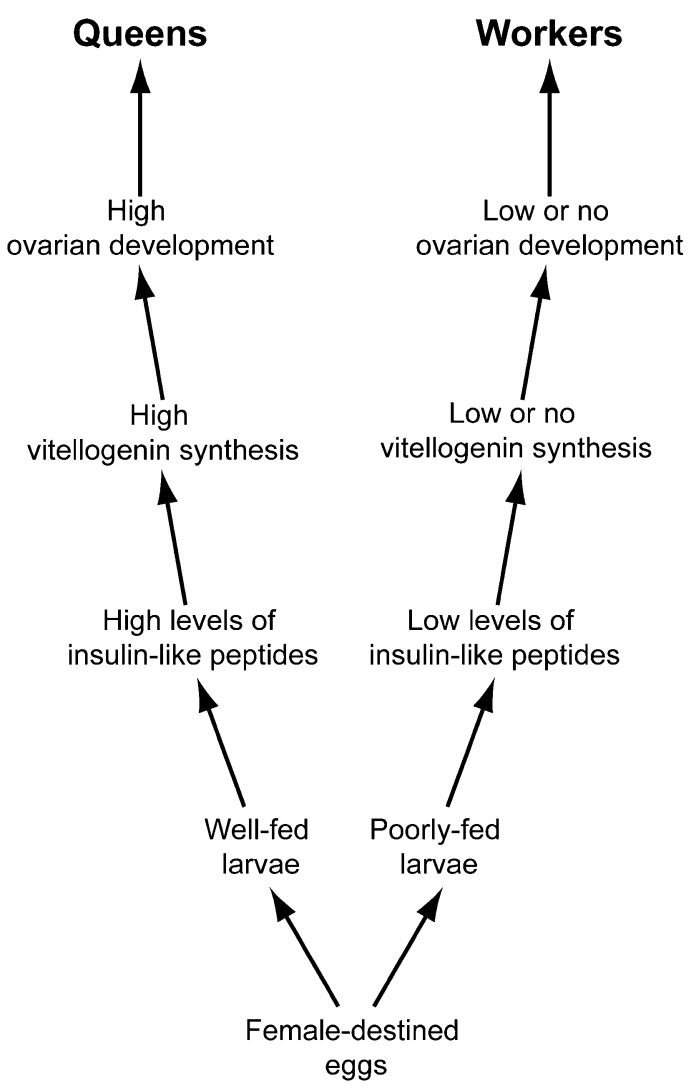

Cooperation in Social Insects, Fig. 2 Our current understanding of the proximate mechanism of reproductive caste differentiation

number of offspring)? This can be split up into several sub-questions such as why does natural selection favor group living instead of solitary living; why is the division of labor, the partitioning of tasks between different individuals, more efficient than each individual performing tasks independent of each other; and how does natural selection produce elaborate communications systems such as the dance language of honey bees, for example? (Gadagkar 1997).

We now have a fairly sophisticated understanding of the proximate mechanisms of social behavior and cooperation. These mechanisms may vary between the primitively and highly eusocial species. In primitively eusocial species, queens and workers are not morphologically differentiated. Queens and workers and sometimes even the sub-groups of workers that specialize in performing different tasks are referred to as castes. Caste differentiation in the highly eusocial species takes place in their early larval stages leading to the eclosion of differentiated adults that can no longer change their fate. In the primitively eusocial species, however, adults at eclosion are completely or nearly totipotent and can subsequently differentiate into queens or workers. Since the main difference between queens and workers is that of reproduction versus sterility, caste differentiation in highly eusocial species is developmentally regulated, while in primitively eusocial species, it is socially regulated. In highly eusocial species, the starting point of such differentiation is differential nutrition between queen-destined larvae and workerdestined larvae. The difference in nutrition may be one of the quality of the food, as in the case of the honey bee where queen larvae are fed with a sugar-rich royal jelly, while workers' larvae are fed with ordinary worker jelly. Or it may involve a difference in the quantity of food such that better-fed larvae become queens and poorly fed larvae become workers. Either way, differential nutrition channels larvae into alternate developmental pathways such that better-nourished larvae produce more insulinlike peptides, turn on vitellogenin synthesis, develop their ovaries, and become queens. Conversely, poorly nourished individuals produce less insulin-like peptides, produce little or no vitellogenin, fail to develop their ovaries, and become sterile workers (Chandra et al. 2018) (Fig. 2). Further subdivision of workers into sub-castes can also be mediated by differential nutrition. In primitively eusocial species where caste differentiation is socially regulated, behavioral dominance-subordinate interactions 
modulate caste differentiation such that behaviorally dominant individuals become egg-laying queens and subordinate individuals become nonegg-laying workers. Further subdivision among workers into intranidal workers (those who work inside the nest) versus extranidal workers (those who work outside the nest) can also be mediated by similar aggressive interactions such that the relatively more dominant individuals among the workers, work at home, and the least dominant individuals work outside, foraging for food and building material required by the colony.

Social insects accomplish many rather complex tasks both inside and outside their nests that appear to be inconsistent with their small brains and limited neural capacity. The secret of their success in achieving complex outcomes incommensurate with the cognitive capacities of individual insects is that they use a process of decentralized self-organization - large numbers of individuals follow simple local rules, but there emerges a complex outcome due to their collective behavior. This is sometimes called social intelligence and is the subject of intense investigations by interdisciplinary teams of biologists, physicists, and mathematicians (Camazine et al. 2003).

Understanding the ultimate causation of sociality, namely, the evolution by natural selection of cooperation and altruism, has been an even greater preoccupation of social insect researchers. The paradox of altruism referred to above was largely solved, at least in theory, by the realization that evolutionary fitness can be gained not only by producing one's own offspring but also by aiding the reproduction of genetic relatives. Indeed, one can combine the two kinds of fitness, direct (through offspring production) and indirect (through aiding genetic relatives), in a simple additive manner. The sum of these two kinds of fitness is known as inclusive fitness. Hamilton's rule predicts that natural selection can promote the evolution of altruistic behavior when the benefit to the recipient of altruism multiplied by the proportion of genes shared between the altruist and the recipient is greater than the cost incurred by the altruist (Box 1). This formulation of the solution to the paradox of altruism is known by several names - inclusive fitness theory, Hamilton's rule, or kin selection. There has been a great deal of effort to develop a sound mathematical foundation of this theory and some (but not enough) effort to see if animals actually behave as if they obey Hamilton's rule. Hamilton's rule can guide empirical researchers in the field and in the laboratory to ask the right questions and measure the right quantities. Nevertheless, very few studies have adequately measured all three parameters in Hamilton's rule to pronounce a clear verdict on whether altruists always obey Hamilton's rule or whether they sometimes get away without obeying the rule. The latter possibility suggests that there may be other ways of solving the altruism paradox. Selection at the level of the individual and also at the level of the whole colony (known as multilevel selection) is sometimes considered as promising avenues for future research (Gadau and Fewell 2009). Meanwhile, the very validity of the inclusive fitness approach is being questioned by some prominent researchers, leading to a bitter controversy (Nowak et al. 2010). As a result, the study of cooperation in insect societies has gained a new vibrant dynamism, attracting much interest from theoreticians and empiricists alike, well beyond the traditional social insect community. Although many interesting phenomena have been discovered and their evolutionary logic is being worked out, many more phenomena surely await discovery. The study of cooperation in social insects is worthy of attention from practitioners of diverse disciplines, not only physics, chemistry, mathematics, and engineering in the natural sciences but also psychology, sociology, political science, and related disciplines in the social sciences. 


\section{Box 1 Hamilton's Rule}

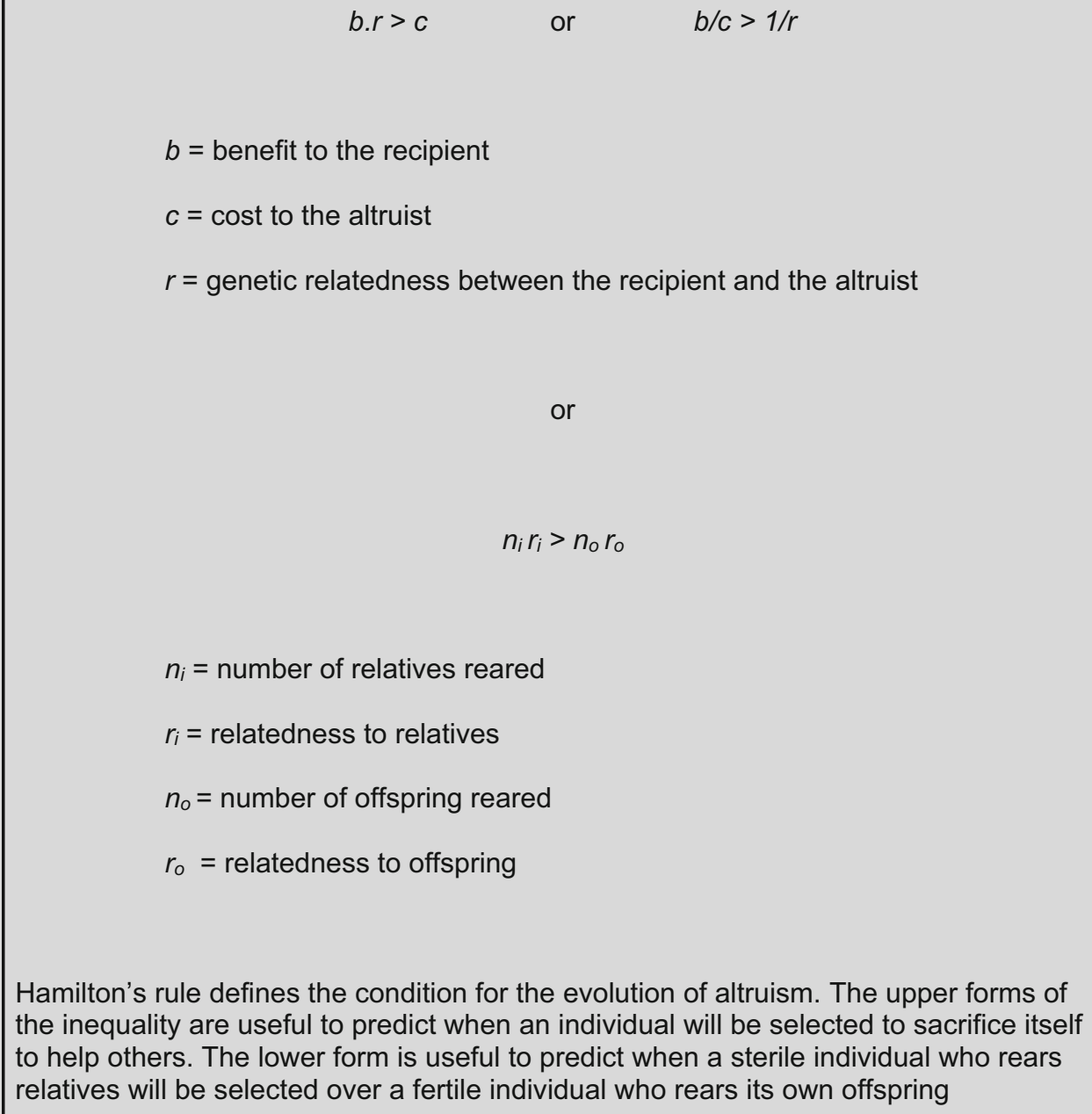

Hamilton's rule defines the condition for the evolution of altruism. The upper forms of the inequality are useful to predict when an individual will be selected to sacrifice itself to help others. The lower form is useful to predict when a sterile individual who rears relatives will be selected over a fertile individual who rears its own offspring

\section{References}

Camazine, S., Deneubourg, J.-L., Franks, N. R., Sneyd, J., Theraulaz, G., \& Bonabeau, E. (2003). Selforganization in biological systems. Princeton/Oxford: Princeton University Press.

Chandra, V., Fetter-Pruneda, I., Oxley, P. R., Ritger, A. L., McKenzie, S. K., Libbrecht, R., \& Kronauer, D. J. C. (2018). Social regulation of insulin signaling and the evolution of eusociality in ants. Science, 361, 398-402.

Gadagkar, R. (1997). Survival strategies: Cooperation and conflict in animal societies. Cambridge, MA/Hyderabad: Harvard University Press/Universities Press.

Gadagkar, R. (2001). The social biology of Ropalidia marginata: Toward understanding the evolution of Eusociality. Cambridge, MA: Harvard University Press. 
Gadau, J., \& Fewell, J. (Eds.). (2009). Organization of insect societies - From genome to sociocomplexity. Cambridge, MA: Harvard University Press.

Hölldobler, B., \& Wilson, E. O. (1990). The ants. Cambridge, MA: Belknap Press of Harvard University Press.

Korb, B., \& Thorne, B. (2017). Sociality in termites. In D. R. Rubenstein \& P. Abbot (Eds.), Comparative social evolution (pp. 124-153). Cambridge: Cambridge University Press.

Mandal, S. (2018). How do animals find their way back home? A brief overview of homing behavior with special reference to social Hymenoptera. Insectes Sociaux, 65, 521-536. https://doi.org/10.1007/s00040018-0647-2.
Nowak, M. A., Tarnita, C. E., \& Wilson, E. O. (2010). The evolution of eusociality. Nature, 466, 1057-1062. https://doi.org/10.1038/nature09205.

Page, R. E., Jr. (2013). The spirit of the hive - The mechanisms of social evolution. Cambridge, MA: Harvard University Press.

Rubenstein, D. R., \& Abbot, P. (Eds.). (2017). Comparative social evolution. New York: Cambridge University Press.

Seeley, T. D. (2010). Honeybee democracy. Princeton/ Oxford: Princeton University Press.

Wehner, R. (1992). Arthropods. In F. Papi (Ed.), Animal homing (pp. 45-144). London: Chapman and Hall.

Wilson, E. O. (1971). The insect societies. Cambridge, MA: Harvard University Press.

Winston, M. L. (1987). The biology of the honey bee. Cambridge, MA: Harvard University Press. 